\title{
Fractional heat shock protein 27 urine excretion as a short-term predictor in acute exacerbation of chronic obstructive pulmonary disease
}

\author{
Denise Traxler ${ }^{1,2} \wedge$, Matthias Zimmermann ${ }^{2,3} \wedge$, Elisabeth Simader ${ }^{2,4}$, Elisa Einwallner ${ }^{5}$, \\ Dragan Copic $^{6} \wedge$, Alexandra Graf ${ }^{7} \wedge$, Thomas Mueller ${ }^{8}$, Cecilia Veraar $^{9} \wedge$, Mitja Lainscak ${ }^{10,11}$, Robert Marčun ${ }^{12} \wedge$, \\ Mitja Košnik $^{12}$, Matjaž Fležar ${ }^{12}$, Aleš Rozman $^{12}$, Peter Korošec ${ }^{12}$, Walter Klepetko ${ }^{6}$, Bernhard Moser $^{6} \wedge$, \\ Hendrik J. Ankersmit ${ }^{2,6 \wedge}$
}

${ }^{1}$ Division of Cardiology, Department of Internal Medicine II, Medical University of Vienna, Vienna, Austria; ${ }^{2}$ Christian Doppler Laboratory for Cardiac and Thoracic Diagnosis and Regeneration, Medical University of Vienna, Austria; ${ }^{3}$ Department of Oral and Maxillofacial Surgery, Medical University of Vienna, Austria; ${ }^{4}$ Division of Rheumatology, Department of Internal Medicine III, Medical University of Vienna, Austria; ${ }^{5}$ Department of Laboratory Medicine, Medical University Vienna, Vienna, Austria; ${ }^{6}$ Division of Thoracic Surgery, Department of Surgery, Medical University of Vienna, Austria; ${ }^{7}$ Center for Medical Statistics, Informatics and Intelligent Systems, Medical University of Vienna, Vienna, Austria; ${ }^{8}$ Department of Clinical Pathology, Hospital of Bolzano, Bolzano, Italy; ${ }^{9}$ Division of Cardiothoracic and Vascular Anesthesia and Intensive Care Medicine, Department of Anesthesia, Intensive Care Medicine, and Pain Medicine, Medical University of Vienna, Vienna, Austria; ${ }^{10}$ Division of Cardiology, General Hospital Murska Sobota, Murska Sobota, Slovenia; ${ }^{11}$ Faculty of Medicine, University of Ljubljana, Slovenia; ${ }^{12}$ University Clinic of Pulmonary and Allergic Diseases Golnik, Golnik, Slovenia

Contributions: (I) Conception and design: D Traxler, M Zimmermann, T Mueller, M. Lainscak, B Moser, HJ Ankersmit; (II) Administrative support: D Traxler, E Simader, E Einwallner, D Copic, C Veraar; (III) Provision of study materials or patients: E Einwallner, M Lainscak, M Košnik, M Fležar, A Rozmann, P Korošec, HJ Ankersmit; (IV) Collection and assembly of data: D Traxler, M Zimmermann, E Simader, D Copic, C Veraar; (V) Data analysis and interpretation: D Traxler, M Zimmermann, T Mueller, HJ Ankersmit; (VI) Manuscript writing: All authors; (VII) Final approval of manuscript: All authors.

Correspondence to: Hendrik J. Ankersmit. Division of Thoracic Surgery, Department of Surgery, Medical University of Vienna, Waehringer Guertel 18-20, 1090 Vienna, Austria. Email: hendrik.ankersmit@meduniwien.ac.at.

Background: Chronic obstructive pulmonary disease (COPD) is a major cause of morbidity and mortality and is characterized by episodes of acute exacerbations. Finding a systemic biomarker that reliably predicts outcome after an acute exacerbation remains a major challenge. Heat shock protein 27 (HSP27) has been previously studied in COPD, however, urine excretion trajectory and prognostic value after an exacerbation is unknown.

Methods: In this retrospective post hoc analysis of a prospective study that included 253 COPD patients who were hospitalized for acute exacerbation, 207 patients were analyzed. Urine and serum were sampled at admission, discharge, and 180 days after discharge; urine excretion trajectory was analyzed and correlated with clinicopathological and survival data.

Results: HSP27 urine excretion increased after an exacerbation episode $[1.8 \%$ admission, $1.8 \%$ discharge, $2.3 \% 180$ days after discharge $(\mathrm{P}=0.091)]$. In severely ill patients (GOLD IV) this course was even more distinct [1.6\% admission, $2.1 \%$ discharge, $2.8 \% 180$ days after discharge $(\mathrm{P}=0.007)]$. Furthermore, fractional HSP27 urine excretion at discharge was increased in GOLD IV patients ( $\mathrm{P}=0.031)$. In Kaplan-Meier and

\footnotetext{
^, ORCID: Denise Traxler, 0000-0001-8679-1525; Matthias Zimmermann, 0000-0002-4610-7853; Dragan Copic, 0000-0002-1005-2985; Alexandra Graf, 0000-0003-0035-2658; Cecilia Veraar, 0000-0001-9378-5000; Robert Marčun, 0000-0001-5129-9186; Mitja Košnik, 0000-0002-4701-7374; Peter Korošec, 0000-0002-0835-1599; Bernhard Moser, 0000-0002-6037-5475; Hendrik J. Ankersmit, 0000-0002$8761-3517$.
} 
univariable Cox proportional hazard models patients with HSP27 urine excretion below $0.845 \%$ showed significantly worse survival at 30,90 and 180 days after discharge. In a multivariable Cox proportional hazard model including established COPD outcome parameters fractional HSP27 urine excretion remained a significant predictor of survival at 30 and 90 days after discharge. Comparing this model to our already published model that includes HSP27 serum concentration we could show that fractional HSP27 urine excretion performs better in short-term survival.

Conclusions: Our findings provide novel information about fractional HSP27 urine excretion trajectory in acute exacerbation of COPD. Fractional HSP27 urine excretion may be significantly reduced during an episode of acute exacerbation in COPD patients and may be used as a predictor of short-term all-cause mortality.

Keywords: Biomarkers; heat shock protein 27 heat-shock proteins (HSP27 heat-shock proteins); pulmonary disease; chronic obstructive; urine

Submitted May 04, 2020. Accepted for publication Sep 30, 2020.

doi: 10.21037/atm-20-3683

View this article at: http://dx.doi.org/10.21037/atm-20-3683

\section{Introduction}

Chronic obstructive pulmonary disease (COPD) is the fourth leading cause of death worldwide (1). It is characterized by acute exacerbations (AECOPD) that significantly affect morbidity and mortality both short- and longterm after an exacerbation episode (2). AECOPD, defined as acute worsening of respiratory symptoms associated with physiological deterioration beyond day-to-day variations, is an event in the natural disease progression (3). Hitherto COPD has been understood as a pulmonary disease that is characterized by structural abnormalities of airways and pulmonary parenchyma. However, recent studies indicate systemic inflammatory reactions to be associated with these pathologies. T cell mediated autoimmunity has been suggested in COPD patients. An increased number of CD2 $8^{\text {null }} \mathrm{CD} 8^{+}$and $\mathrm{CD} 28^{\text {null }} \mathrm{CD} 4^{+}$ has been reported in COPD and this $\mathrm{T}$ cell senescence is associated with the loss of HSP90 (4-6). As a consequence, this immune activation can also be detected systemically by elevation of inflammatory mediators (7).

Diagnosis and monitoring of COPD patients is mainly performed using spirometry, however, in acute exacerbation this method may not be recommended as it is too difficult to perform (8). Apart from a variety of clinical parameters (9-11) several blood biomarkers e.g., procalcitonin (12), CRP (13), uric acid (14), cardiac troponin T (cTnT) and $\mathrm{N}$-terminal pro-brain natriuretic protein (NT-proBNP) (15) have been studied in AECOPD. Finding a systemic marker that offers a prognostic value is of enormous importance as identifying COPD patients at high risk of poor outcome could help intensifying interventions to improve quality of life and reduce morbidity in those patients. However, identifying such a biomarker is difficult, as no gold standard to definitively clarify its value has been established, yet.

Heat shock proteins (HSPs) are a group of highly preserved stress proteins that are usually found intracellularly. As part of the intracellular stress response HSPs are released from cells to interact with adjacent cells or may enter the blood stream. They are directly involved in chaperoning and regulating cell death pathways (16). Heat shock protein 27 (HSP27) is a member of the small HSP family with a molecular weight of approximately $27 \mathrm{kDa}$. It is involved in a multitude of cellular functions such as stress tolerance, protein degradation and cell death (17). It further provides protective mechanisms against oxidativeinflammatory conditions (18). Extracellular HSP27 can act anti-inflammatory by increasing the level of the antiinflammatory cytokine interleukin-10 and attenuating migration and adhesion of macrophages $(19,20)$. It is thought to exert an important role in many different conditions such as cancer and cardiovascular disease $(21,22)$. Its role as a diagnostic and prognostic marker in COPD has already been studied extensively with partly contradictory results (23-28).

As a consequence of observations on the expression of HSP27 in COPD a possible role of this protein as a useful marker also in episodes of acute exacerbation is suggested as HSP27 is released from PBMCs (29). Previous work 
on HSP27 in serum and plasma included only a small set of patients. Recently published data showed increased serum concentration during hospitalization for an acute exacerbation, further elevated serum HSP27 was associated with worse survival (28). Nonetheless no data are available on fractional HSP27 urine excretion in AECOPD. We hypothesize that fractional HSP27 urine excretion might provide prognostic value in those patients. To test this hypothesis, we performed serum and urine measurements and correlated them with clinicopathological and survival data.

We present the following article in accordance with the STROBE reporting checklist (available at http://dx.doi. org/10.21037/atm-20-3683).

\section{Methods}

\section{Patient population}

The study has been approved by the National Ethics Committee of the Republic of Slovenia (EC number: $65 / 02 / 09)$, was conducted according to the declaration of Helsinki (as revised in 2013) and is registered at ClinicalTrials.gov (NCT01225627). Informed consent was provided by all participants. Study protocol and main findings were published previously $(30,31)$. In brief, 253 patients that were admitted for AECOPD between 2009 and 2011 at the University Clinic of Pulmonary and Allergic Diseases Golnik, Slovenia were prospectively enrolled. After informed consent was given, patients were screened for inclusion and exclusion criteria as described previously (30) and enrolled according to their eligibility. Blood and urine samples were collected at admission, discharge, and 180 days after discharge. A post hoc analysis of 207 patients was performed, as samples were not available for 28 patients and 18 patients were excluded from the final analyses due to missing values. HSP27 serum levels have recently been analyzed in this patient cohort (28), in this re-analysis four patients were excluded due to missing urine samples. 3 -year-mortality data (all cause) were available for all patients. Blood was taken at admission for full blood count, CRP, cTnT, NT-proBNP and other measurements as per good clinical practice.

\section{Quantification of urine HSP27}

A commercially available ELISA Kit (DYC1580, R\&D Systems, Minneapolis, MN, USA) was used to assess serum and urine total HSP27 concentrations according to the manufacturer's protocol. This kit was previously compared to other commercial kits and showed superior performance of diagnostic accuracy in diagnosis of pathologies of the lung (32). Inter- and intraassay variability for urine samples of this ELISA kit has been previously assessed (33).

Fractional HSP27 excretion was calculated as previously described (33) with the formula: [(urine HSP27 $\times$ serum creatinine $) /($ serum HSP $27 \times$ urine creatinine $) \times 100$ to eliminate varying water excretion and resorption as confounding factors as well as to compensate the influence of comprised renal capillary endothelial permeability during exacerbation (34).

\section{HSP27 cut off determination}

Cut off values of HSP27 urine excretion at admission for Kaplan-Meier curve analysis and Cox proportional hazard models for all cause mortality calculations were identified using Cutoff Finder version 2.1, a freely available R functions based web application (35).

\section{Statistics}

Data obtained were evaluated statistically using IBM SPSS Statistics version 23 (SPSS Inc., Chicago, USA) and GraphPad Prism 6 software (GraphPad Software Inc., LA Jolla, CA, USA). Mann-Whitney U test and KruskalWallis test with each pairwise $\mathrm{P}$ value correction were used to compare non-parametric, unpaired variables and Friedman test with each pairwise $\mathrm{P}$ value correction was used to compare non-parametric paired variables and expressed as median and interquartile range (IQR). KaplanMeier curves and log-rank test were used to evaluate eventfree (time to death) interval. Cox proportional hazard models were built to evaluate prognostic significance of established factors and fractional HSP27 urine excretion at 30 days, 90 days, 180 days, 1 year and 3 years and were expressed as hazard ratio (HR) with corresponding $95 \%$ confidence intervals (CI). As this is an explorative reanalysis of an already published dataset, we aimed to compare this model to the published one (HSP27 serum concentration as a prognostic predictor) (28). Harrell's c-Index was assessed for both models at all above given time points. Binary logistic regression was performed to obtain predicted probability values of the models and ROC curves with those values were plotted. Area under the curve (AUC) was used to compare both models at all time 


\section{Page 4 of 13}

points. All tests were performed in a two-sided manner. $\mathrm{P}$ values equal or below 0.05 were considered as statistically significant.

\section{Results}

\section{Study population}

All 207 patients were included into this explorative reanalysis. HSP27 urine excretion values were available at admission of 207 patients, at discharge of 189 patients and at 180 days after discharge of 107 patients. A summary of clinicopathological parameters of all included patients from admission is depicted in Table 1. Patients are predominately male $(72 \%)$ and suffered from advanced COPD (GOLD II-IV). Median age was 72 years (IQR: 64-77). All patients received COPD specific optimal medical therapy. Follow up time was 1095 days, the number of deaths at 30 days after discharge was $7(3.4 \%), 13(6.3 \%)$ at 90 days, $25(12.1 \%)$ at 180 days, $70(33.8 \%)$ at 2 years and $98(47.3 \%)$ at 3 years.

\section{HSP27 urine excretion in an episode of acute exacerbation in COPD}

Both HSP27 urine and serum concentration were measured at admission, discharge, and 180 days after discharge. Both showed decreasing values during the observed time frame. HSP27 urine concentration (median) was $3,300.6 \mathrm{pg} / \mathrm{mL}$ (IQR: 2,160.1-4,939.2 $\mathrm{pg} / \mathrm{mL}$ ) at admission, $2,857.3 \mathrm{pg} / \mathrm{mL}$ (IQR: $1,491.9-4,783.1 \mathrm{pg} / \mathrm{mL}$ ) at discharge and $2,533.9 \mathrm{pg} / \mathrm{mL}$ (IQR: $1,557.4-4,836.8 \mathrm{pg} / \mathrm{mL}$ ) 180 days after discharge $(\mathrm{P}=0.091$, Figure 1A). HSP27 serum concentration (median) was $2,204.0 \mathrm{pg} / \mathrm{mL}$ (IQR: $1,545.7-3,013.1 \mathrm{pg} / \mathrm{mL}$ ) at admission, $1,993.6 \mathrm{pg} / \mathrm{mL}$ (IQR: 1,504.4-2,686.5 pg/mL) at discharge and 1,635.6 pg/mL (IQR: 1,333.5-2,405.2 pg/mL) 180 days after discharge $(\mathrm{P}=0.101$, Figure $1 A)$. Serum and urine concentration of HSP27 did not significantly correlate at admission $(\mathrm{r}=-0.05, \mathrm{P}=0.471)$.

Levels of proteins in urine are highly dependent on individually varying water excretion and resorption as well as renal capillary endothelial permeability during an episode of exacerbation. To eliminate those confounding factors fractional urine excretion of HSP27 was calculated. Fractional HSP27 urine excretion exhibited a trend towards significantly decreased values during and immediately after an episode of acute exacerbation. Fractional HSP27 urine excretion (median) was $1.8 \%$ (IQR: $1.0-4.1 \%$ ) at admission, 1.8\% (IQR: $1.1-3.9 \%$ ) at discharge and $2.3 \%$ (OQR:
1.4-4.7\%) 180 days after discharge $(\mathrm{P}=0.091$, Figure $1 A)$. Further admission serum HSP27 and fractional HSP27 urine excretion correlated significantly $(\mathrm{r}=-0.241, \mathrm{P}<0.001)$.

If only severely ill patients (GOLD IV) are included, a more distinct course could be demonstrated. Fractional HSP27 urine excretion (median) in GOLD IV patients was 1.6\% (IQR: $1.0-3.2 \%$ ) at admission, $2.1 \%$ (IQR: 1.1-5.2\%) at discharge and $2.8 \%$ (IQR: 1.6-6.6\%) 180 days after discharge $(\mathrm{P}=0.007$, Figure $1 B)$. A statistical difference could be detected between admission and 180 days after discharge $(\mathrm{P}=0.007)$. HSP27 serum and urine concentration did not differ in GOLD IV patients (Figure 1B).

As serum HSP27 allows discrimination between mild and severe COPD (24), but no data on fractional HSP27 urine excretion in different GOLD stages is available we compared HSP27 urine excretion between GOLD stages. Fractional HSP27 urine excretion was elevated in GOLD IV patients after an episode of acute exacerbation (GOLD II vs. GOLD IV, $\mathrm{P}=0.031)$ and 180 days after admission (GOLD III vs. GOLD IV, $\mathrm{P}=0.035$ ), however not at admission ( $\mathrm{P}=0.819)$. However, HSP27 urine excretion did not correlate significantly with age at any time point.

By comparing fractional HSP27 urine excretion, serum, and urine HSP27 and eGFR in decedents and survivors at 180 days after admission we observed significantly increased serum HSP27 levels in decedents (Figure 1C).

\section{Fractional HSP27 urine excretion at admission as a predictor of survival}

To assess admission fractional HSP27 urine excretion's impact as a biomarker that predicts all-cause mortality we performed Kaplan Meier curve analyses and built a Cox proportional hazard model. Survival data were available for all patients for up to 3 years after admission. Using the $\mathrm{R}$ functions-based web application Cutoff Finder 2.1 we could identify a cut off level for admission fractional HSP27 urine excretion that predicts survival within the first 180 days after discharge $(0.845 \%)$. We further compared HSP27 to the well-known biomarkers NT-proBNP (Figure $2 A$ ) and cTnT (Figure 2B). Median survival time for patients with values below the cut off was 1309 days (95\% CI: 806-1,812 days) and for patients with values above the cut off was 1185 days (95\% CI: 943-1,427 days). Patients with fractional HSP27 urine excretion at admission below or equal $0.845 \%$ had significantly worse survival 30 days $(\mathrm{P}=0.007, \mathrm{Log}$ rank $=7.292), 90$ days $(\mathrm{P}=0.006$, Log rank $=7.608)$ and 180 days $(\mathrm{P}=0.043, \log$ rank $=4.100)$ after discharge (Figure $2 C)$. 
Table 1 Patients' baseline demographic and laboratory values at admission

\begin{tabular}{|c|c|c|c|c|c|c|c|}
\hline Baseline & $\begin{array}{l}\text { All patients, } \\
\qquad n=207\end{array}$ & $\begin{array}{c}\text { HSP27 high } \\
(>0.854 \%), n=169\end{array}$ & $\begin{array}{c}\text { HSP27 low } \\
(\leq 0.854 \%), n=38\end{array}$ & $P$ value & $\begin{array}{c}\text { Survivors at } \\
180 \text { days, } n=186\end{array}$ & $\begin{array}{c}\text { Decedents at } \\
180 \text { days, } n=26\end{array}$ & $P$ value \\
\hline Gender (male/female) & $\begin{array}{c}148(72 \%) / 59 \\
(28 \%)\end{array}$ & $\begin{array}{c}113 \\
(70 \%) / 56(33 \%)\end{array}$ & $35(92 \%) / 3(8 \%)$ & 0.002 & $\begin{array}{c}130 \\
(71 \%) / 52(29 \%)\end{array}$ & $18(72 \%) / 7(28 \%)$ & 0.953 \\
\hline Age (years; median \pm IQR) & $72.4(63.5-77.3)$ & $71.9(63.4-77.1)$ & $74.4(66.0-78.4)$ & 0.284 & $71.2(62.8-76.9)$ & 76.1 (71.2-81.2) & 0.009 \\
\hline GOLD & & & & 0.491 & & & 0.064 \\
\hline GOLD III & $93(45 \%)$ & $73(43 \%)$ & $20(53 \%)$ & & $85(47 \%)$ & $8(32 \%)$ & \\
\hline GOLD IV & $89(43 \%)$ & $74(44 \%)$ & $15(39 \%)$ & & $73(40 \%)$ & $16(64 \%)$ & \\
\hline $\mathrm{BMI}\left(\mathrm{kg} / \mathrm{m}^{2} ;\right.$ median $\left.\pm \mathrm{IQR}\right)$ & $25.7(23.3-30.4)$ & $25.0(23.3-30.3)$ & $28.0(23.2-32.5)$ & 0.401 & $25.8(23.4-30.4)$ & $24.2(21.2-30.0)$ & 0.252 \\
\hline Heart failure & $54(26 \%)$ & $43(25 \%)$ & $11(29 \%)$ & 0.657 & $43(24 \%)$ & $11(44 \%)$ & 0.030 \\
\hline Arterial fibrillation & $19(16 \%)$ & $14(16 \%)$ & $5(16 \%)$ & 0.985 & $17(17 \%)$ & $2(13 \%)$ & 0.662 \\
\hline Ischemic heart disease & $17(15 \%)$ & $13(15 \%)$ & $4(13 \%)$ & 0.764 & $14(14 \%)$ & $3(19 \%)$ & 0.606 \\
\hline Arterial hypertension & $51(44 \%)$ & $35(41 \%)$ & $5(16 \%)$ & 0.293 & $44(44 \%)$ & $7(44 \%)$ & 0.989 \\
\hline Diabetes mellitus & $27(23 \%)$ & $20(23 \%)$ & $7(23 \%)$ & 0.939 & $23(23 \%)$ & $4(25 \%)$ & 0.844 \\
\hline $\mathrm{CRP}(\mathrm{mg} / \mathrm{L}$, median $\pm \mathrm{IQR})$ & $23.1(3.8-77.2)$ & $22.3(4.2-87.6)$ & $32.7(3.4-67.3)$ & 0.988 & $21.5(3.7-75.6)$ & $37.9(14.0-77.2)$ & 0.312 \\
\hline cTnT (ng/L, median \pm IQR) & $0.0(0.0-0.017)$ & $0.0(0.0-0.015)$ & $0.0(0.0-0.029)$ & 0.854 & $0.0(0.0-0.015)$ & $0.0(0.0-0.03)$ & 0.140 \\
\hline $\begin{array}{l}\text { Urea-admission }(\mathrm{mmol} / \mathrm{L} \text {, } \\
\text { median } \pm \mathrm{IQR})\end{array}$ & $6.9(5.3-9.7)$ & $6.7(5.3-9.7)$ & $7.35(5.4-9.6)$ & 0.613 & $6.7(5.3-9.7)$ & $7.5(5.7-9.2)$ & 0.429 \\
\hline $\begin{array}{l}\text { eGFR }(C D K-E P I)(\mathrm{mL} / \mathrm{min} / 1.73 \\
\left.\mathrm{m}^{2}, \text { median } \pm \mathrm{IQR}\right)\end{array}$ & $81.7(65.2-93.5)$ & 81.7 (64.9-93.6) & $83.1(72.5-91.3)$ & 0.767 & $82.3(66.8-93.3)$ & $76.0(63.6-93.5)$ & 0.667 \\
\hline $\begin{array}{l}\text { HSP27 urine excretion: } \\
\text { admission }(\%, \text { median } \pm \text { IQR) }\end{array}$ & $1.8(1.0-4.1)$ & $2.2(1.3-5.1)$ & $0.6(0.4-0.7)$ & $<0.001$ & $1.8(1.0-4.4)$ & $1.6(0.8-2.4)$ & 0.145 \\
\hline $\begin{array}{l}\text { HSP27 urine excretion: } \\
\text { discharge (\%, median } \pm \text { IQR) }\end{array}$ & $1.8(1.1-3.9)$ & $2.0(1.2-4.7)$ & $1.2(0.8-1.9)$ & 0.001 & $1.8(1.1-3.9)$ & $1.7(1.1-3.2)$ & 0.606 \\
\hline $\begin{array}{l}\text { HSP27 urine excretion: } \\
180 \text { days after discharge ( } \% \text {, } \\
\text { median } \pm \text { IQR) }\end{array}$ & $2.3(1.4-4.7)$ & $2.7(1.6-5.3)$ & $0.9(0.7-1.8)$ & 0.000 & $2.3(1.4-4.7)$ & - & - \\
\hline $\begin{array}{l}\text { HSP27 serum: admission } \\
(\mathrm{pg} / \mathrm{mL} \text {, median } \pm \mathrm{IQR})\end{array}$ & $\begin{array}{c}2,204.0 \\
(1,545.7-3,013.1)\end{array}$ & $\begin{array}{c}2,030.7 \\
(1,487.9-2,719.2)\end{array}$ & $\begin{array}{c}2,817.6 \\
(2,226.6-3,543.0)\end{array}$ & 0.001 & $\begin{array}{c}2,099.9 \\
(1,521.3-2,802.4)\end{array}$ & $\begin{array}{c}3100.1 \\
(2286.7-3462.6)\end{array}$ & 0.003 \\
\hline
\end{tabular}

Table 1 (continued) 
Table 1 (continued)

\begin{tabular}{|c|c|c|c|c|c|c|c|}
\hline Baseline & $\begin{array}{l}\text { All patients, } \\
\qquad n=207\end{array}$ & $\begin{array}{c}\text { HSP27 high } \\
(>0.854 \%), n=169\end{array}$ & $\begin{array}{c}\text { HSP27 low } \\
(\leq 0.854 \%), n=38\end{array}$ & $P$ value & $\begin{array}{c}\text { Survivors at } \\
180 \text { days, } n=186\end{array}$ & $\begin{array}{c}\text { Decedents at } \\
180 \text { days, } n=26\end{array}$ & $P$ value \\
\hline $\begin{array}{l}\text { HSP27 serum: discharge } \\
(\mathrm{pg} / \mathrm{mL} \text {, median } \pm \mathrm{IQR})\end{array}$ & $\begin{array}{c}1,993.6 \\
(1,504.4-2,686.5)\end{array}$ & $\begin{array}{c}1,948.0 \\
(1,520.4-2,675.5)\end{array}$ & $\begin{array}{c}2,224.9 \\
(1,500.4-2,872.0)\end{array}$ & 0.670 & $\begin{array}{c}1,931.1 \\
(1,500.4-2,675.5)\end{array}$ & $\begin{array}{c}2458.7 \\
(1680.7-2827.5)\end{array}$ & 0.195 \\
\hline $\begin{array}{l}\text { HSP27 serum: } 180 \text { days after } \\
\text { discharge }(\mathrm{pg} / \mathrm{mL} \text {, median } \pm \\
\text { IQR) }\end{array}$ & $\begin{array}{c}1,635.6 \\
(1,333.5-2,405.2)\end{array}$ & $\begin{array}{c}1,653.6 \\
(1,333.5-2,126.8)\end{array}$ & $\begin{array}{c}1,991.4 \\
(1,416.6-2,881.0)\end{array}$ & 0.215 & $\begin{array}{c}1,635.6 \\
(1,333.5-2,405.2)\end{array}$ & - & - \\
\hline $\begin{array}{l}\text { HSP27 urine: discharge } \\
(\mathrm{pg} / \mathrm{mL} \text {, median } \pm \mathrm{IQR})\end{array}$ & $\begin{array}{c}2,857.3 \\
(1,491.9-4,783.1)\end{array}$ & $\begin{array}{c}2,959.5 \\
(1,533.4-5,511.7)\end{array}$ & $\begin{array}{c}1,914.7 \\
(926.6-3,466.1)\end{array}$ & 0.014 & $\begin{array}{c}2,884.1 \\
(1,413.2-4,783.1)\end{array}$ & $\begin{array}{c}2074.8 \\
(1528.3-4979.1)\end{array}$ & 0.720 \\
\hline $\begin{array}{l}\text { HSP27 urine: } 180 \text { days after } \\
\text { discharge }(\mathrm{pg} / \mathrm{mL} \text {, median } \pm \\
\text { IQR) }\end{array}$ & $\begin{array}{c}2,533.9 \\
(1,557.4-4,836.8)\end{array}$ & $\begin{array}{c}2,629.7 \\
(1,670.7-5,340.0)\end{array}$ & $\begin{array}{c}1,764.1 \\
(1,173.9-3,818.2)\end{array}$ & 0.038 & $\begin{array}{c}2,533.9 \\
(1,557.4-4,836.8)\end{array}$ & - & - \\
\hline
\end{tabular}

HSP27, heat shock protein 27; IQR, interquartile range; GOLD, global initiative for chronic obstructive lung disease; BMI, body mass index; LTOT, long-term oxygen therapy; CRP, C-reactive protein; cTnT, cardiac Troponin T; NT-proBNP, n-terminal pro-brain natriuretic peptide.
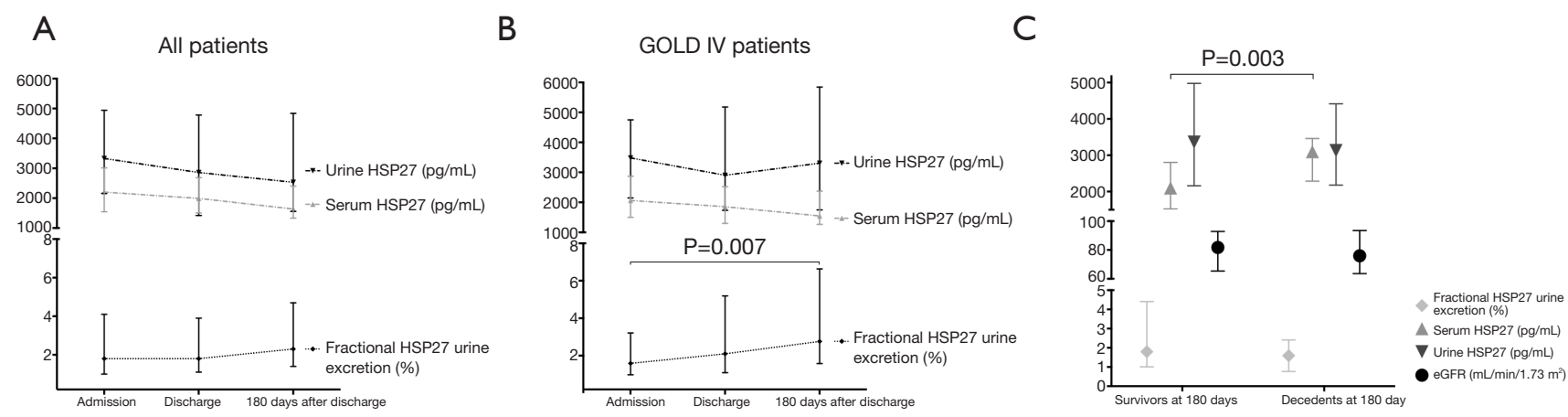

Figure 1 Time course of HSP27 urine and serum concentration as well as fractional HSP27 urine excretion in all patients (A) and GOLD IV patients (B). Differences of HSP27 urine and serum concentration, fractional HSP27 urine excretion and eGFR between survivors and decedents at 180 days after admission (C). HSP27, heat shock protein 27; eGFR, estimated glomerular filtration rate.

There was no significant difference of survival for longer follow up periods ( 1 year: $\mathrm{P}=0.219$, $\log$ rank $=1.511$; 3 years: $\mathrm{P}=0.952, \log$ rank $=0.004)$. Combination of fractional HSP27 urine excretion and NT-proBNP in a Kaplan-Meier survival analysis showed a worse survival in the NT-proBNP-high/HSP27-low group after 180 days (log-rank test $=12.709, \mathrm{P}=0.005)$ (Figure 2D).

Adjusted Cox proportional hazard models were built using established COPD outcome parameters (age, gender, GOLD class, CRP, cTnT and NT-proBNP, Tables 2,3). In a univariable analysis admission fractional HSP27 urine excretion did significantly predict 30 day (HR: 6.1,
95\% CI: 1.4-27.2, Figure 3A), 90 day (HR: 4.1, 95\% CI: 1.4-12.2, Figure $3 B$ ) and 180-day mortality (HR: 2.3, 95\% CI: 1.0-5.4, Figure 3C). For the timepoints 1 year and 3 years HSP27 did not predict mortality (Figure 3D,E). In a multivariable analysis fractional HSP27 urine fraction remained significant at 30 days (HR: 7.1, 95\% CI: $1.6-$ 32.3, Figure $3 A$ ) and 90 days (HR: 4,3, 95\% CI: 1.4-12.8, Figure $3 B$ ) after admission.

We have previously analyzed HSP27 serum concentration as a prognosis predictor in this patient subset and have identified its power in mid-term survival (90 days to 1 year follow-up time) (28). In contrast HSP27 fractional 
A

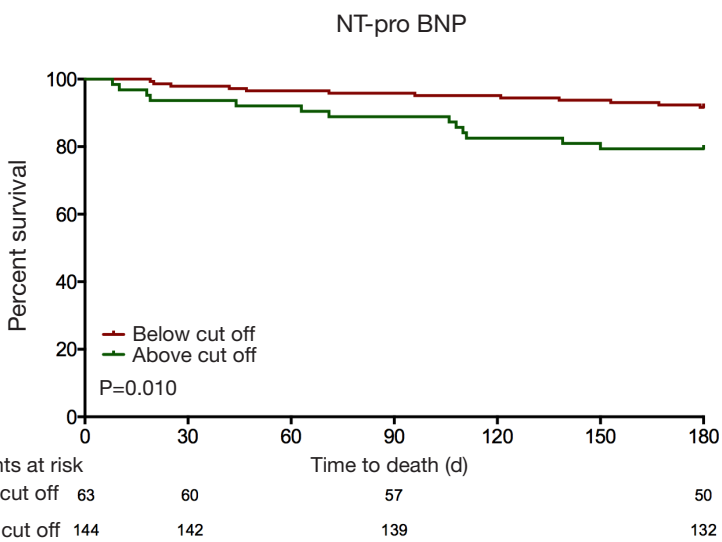

C

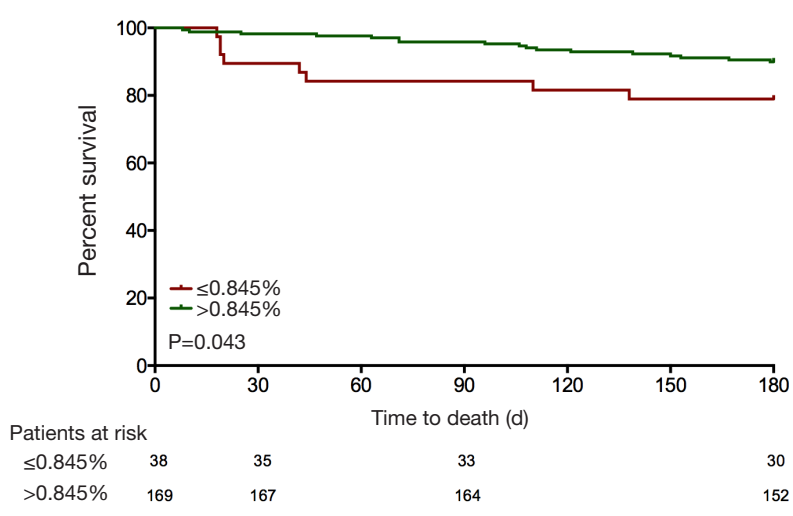

B

cTnT

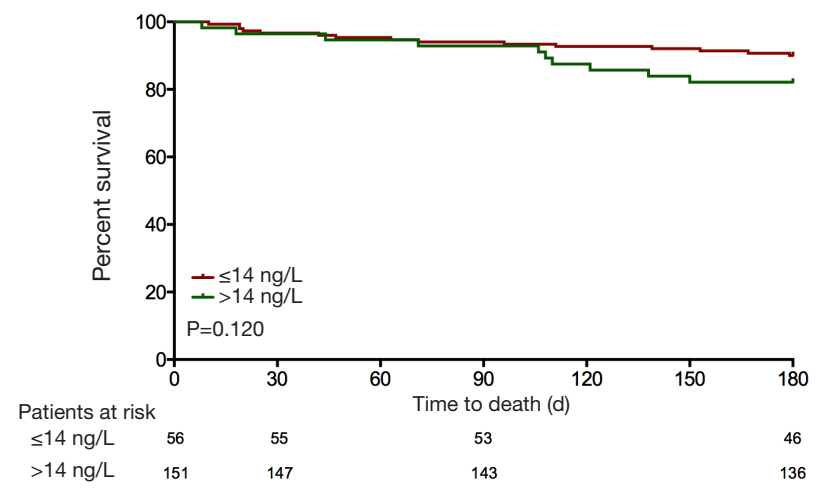

D

HSP27 \& NT-pro BNP

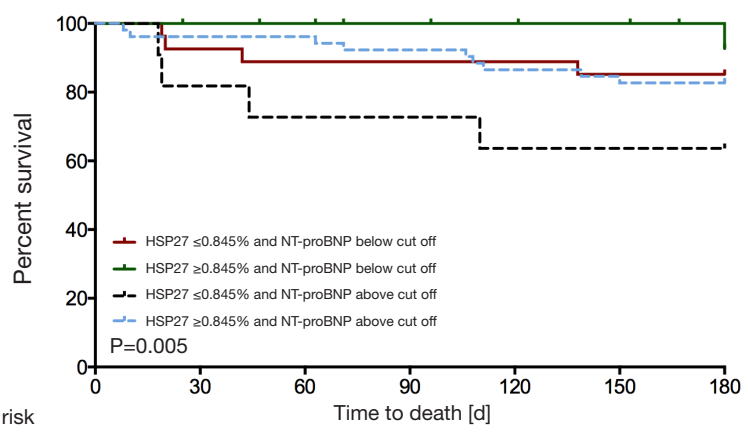

HSP $27 \geq 0.845 \%$

HSP27 $20.845 \%$ \&
NT-proBNP above cut off 52

NT-proBNP above cut off 11

NT-proBNP above cut off
HSP $27 \geq 0.845 \%$ \&

$\begin{array}{ll}\text { NT-proBNP below cut off } & 117 \\ \text { HSP27 }<0.845 \% \& & \\ \text { NT-proBNP below cut off } & 27\end{array}$ $\begin{array}{cc}49 & 43 \\ 9 & 7 \\ 115 & 109 \\ 25 & 23\end{array}$

Figure 2 Kaplan-Meier survival stratified by (A) NT-proBNP, (B) cTnT, (C) HSP27 and (D) combined concentrations of HSP27 and NTproBNP at admission. NT-proBNP, n-terminal pro-brain natriuretic peptide; HSP27, heat shock protein 27; cTnT, cardiac troponin T.

urine excretion seems to reveal its strength in short-term survival (30 days). We compared both Cox proportional hazard models and observed higher c-indices of the models including HSP27 fractional urine excretion compared to the serum model in short-term survival (urine $v s$. serum at 30 days: c-index: 0.850 vs. 0.500 ). This is supported by the AUC of both models (urine vs. serum at 30 days: AUC: 0.701 vs. 0.500). In contrast both c-index and AUC were superior in the serum model on year 1 (Table 4).

\section{Association with other markers}

Increased NT-proBNP concentrations at admission were significantly associated with fatal outcome over the followup period (Figure $2 A$ ), in contrast elevated cTnT levels were not associated with worse outcome (Figure 2B). As CRP, NT-proBNP and cTnT have already been identified as prognostic markers in AECOPD we correlated them with admission fractional HSP27 urine excretion (15,36-40). We did not observe a correlation of admission fractional HSP27 urine excretion with any marker (CRP: $\mathrm{r}=-0.060, \mathrm{P}=0.394$; NT-proBNP: $r=-0.052, \mathrm{P}=0.454$, cTnT: $\mathrm{r}=0.106, \mathrm{P}=0.130$ ).

\section{Discussion}

In the present study we demonstrated decreased factional HSP27 urine excretion at admission due to an episode of acute exacerbation in COPD patients and an increase after 180 days after discharge. This course was even more distinct in advanced COPD patients (GOLD IV). Furthermore, we could identify an admission fractional HSP27 urine excretion cut off value serving as predictors for short-term mortality in Kaplan-Meier curve analyses and a univariable and multivariable Cox proportional hazard model including 


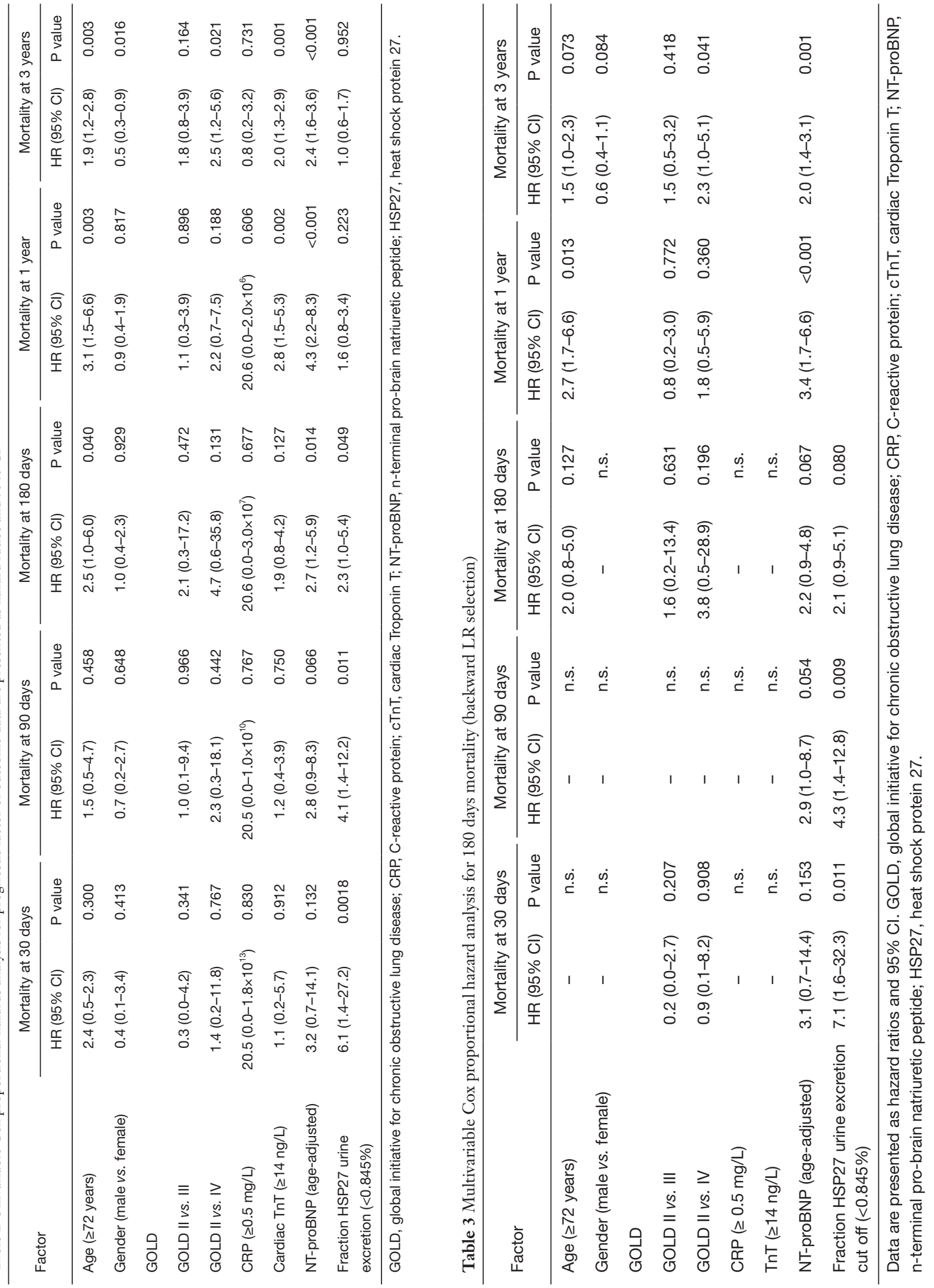


A

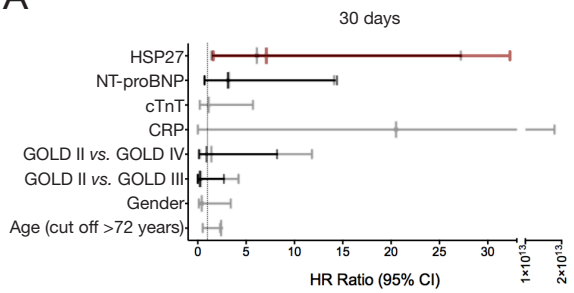

B
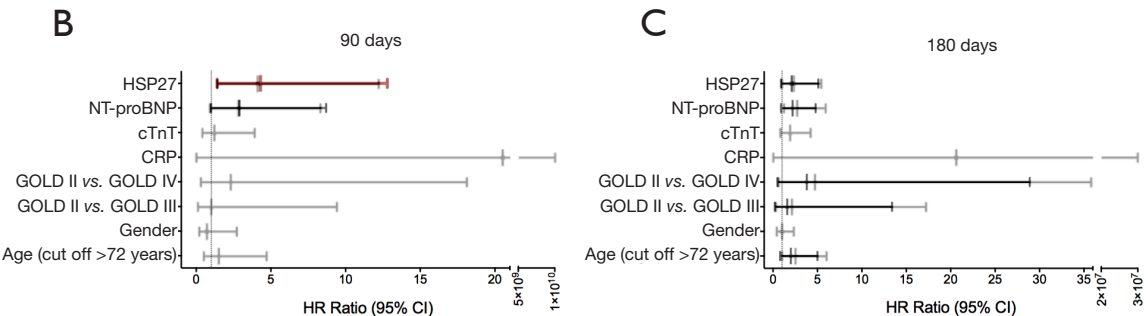

D

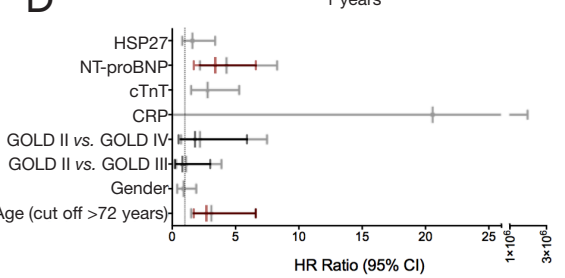

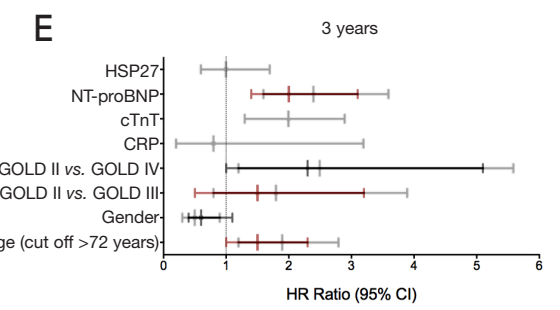

Figure $3 \mathrm{HR}$ and $95 \% \mathrm{CI}$ in univariable (grey bar) and multivariable (black and red bar, for the latter $\mathrm{P}<0.05$ ) Cox regression analysis after 30 days (A), 90 days (B), 180 days (C), 1 year (D) and 3 years (E). Variables remaining in the model after multivariable analyses are presented in red/black. HSP27, heat shock protein 27; NT-proBNP, n-terminal pro-brain natriuretic peptide; cTnT, cardiac troponin T; CRP, C-reactive protein; GOLD, Global Initiative for Chronic Obstructive Lung Disease; HR, hazard ratios.

Table 4 Comparison of c-indices and AUC of the cox proportional hazard model including HSP27 urine excretion and the previously published cox proportional hazard model including HSP27 serum excretion (28)

\begin{tabular}{lcccc}
\hline \multirow{2}{*}{ Time period } & \multicolumn{2}{c}{ HSP27 fractional urine excretion } & \multicolumn{2}{c}{ HSP27 serum excretion } \\
\cline { 2 - 5 } \cline { 2 - 4 } 30 days & C-index & AUC & C-index & AUC \\
90 days & 0.850 & 0.701 & 0.500 & 0.500 \\
180 days & 0.717 & 0.720 & 0.657 & 0.664 \\
1 year & 0.735 & 0.726 & 0.699 & 0.724 \\
3 years & 0.740 & 0.773 & 0.758 & 0.790 \\
\hline
\end{tabular}

AUC, area under the curve; HSP27, heat shock protein 27.

established outcome parameters. Recently published data on HSP27 serum concentration in this patient cohort suggest a role as a predictor of all cause survival for midand long-term follow up (up to 1 year). We hypothesized that fractional HSP27 urine excretion might be a superior prognosis marker especially for the period of 30 days after discharge. Therefore, we compared both models and observed higher Harrell's c-index and AUC in the model including fractional HSP27 urine excretion for 30-day allcause mortality, suggesting a superior performance of the fractional HSP27 urine excretion-based model. However, in 1-year survival analysis the model including HSP27 serum concentration was superior. Survival data up to 3 years were available for our patient cohort. In none of the models HSP27 was a predictor of 3-year mortality, in contrast, we observed NT-proBNP, a well-known cardiac marker, as the main predictor in a multivariable Cox proportional hazard model.

Several previous studies attempted to identify biomarkers that predict clinical outcome after hospitalization for AECOPD. Acute exacerbations are both common and serious conditions in COPD patients and frequency and severity of exacerbation episodes are a major factor limiting survival and quality of life (3). Prevention of exacerbations is generally considered a key strategy in managing COPD (1). Thus, identifying patients who are at high risk for further 


\section{Page 10 of 13}

exacerbations or death is of particular importance (3).

The heat shock response is characterized by increased expression of HSPs due to stress to ensure cell integrity and minimize the extent of apoptosis (17). HSPs can be spilled into the blood system by cell damage to mediate a response by the innate immune system but also by immune cells themselves (29,41). A variety of syndromes e.g., stable COPD (24), sepsis (42) and coronary artery disease (43) are associated with altered HSP27 concentration. Elevated extracellular HSP27 levels are found under inflammatory conditions. Especially oxidative stress induces HSP27 expression. Copper $\left(\mathrm{Cu}^{2+}\right)$, a toxic metal ion that is contained in cigarette smoke, results in increased expression of HSP27. Hence, HSP27 binds $\mathrm{Cu}^{2+}$ and inhibits $\mathrm{Cu}^{2+}$ mediated generation of reactive oxygen species and oxidative stress $(17,44,45)$. Oxidative stress derived from cigarette smoke or air pollutants plays a pivotal role in the pathogenesis of COPD and especially during an exacerbation episode the burden of oxidative stress increases (46). Increased HSP27 expression and extracellular secretion in COPD may reflect mechanisms to protect bronchial epithelial cells from apoptosis under oxidative stress (47). Even though previous studies clearly implicate a role for HSP27 in the pathogenesis of COPD it is still unclear. Increased HSP27 levels in serum of stable COPD patients were demonstrated $(24,48)$ whereas Cui et al. showed decreased plasma HSP27 concentrations but increased lymphocyte HSP27 levels in coal miners suffering from COPD compared to healthy coal miners (25). In another study increased expression of HSP27 in bronchial epithelium of COPD patients was seen (26). In contrast, Cappello et al. could not detect any differences in HSP27 expression in bronchial epithelium of COPD patients and control subjects (27). Elevated intracellular HSP27 expression may result in spillage into the vascular bed. Thus, it is not surprising that we could detect a trend towards increased serum and urine HSP27 concentrations during exacerbation and decreasing concentrations in the stable phase. As HSP27 belongs to the family of small HSPs and is of a size of about $27 \mathrm{kDa}$, renal elimination of HSP27 may be suggested (17). Fractional HSP27 urine excretion reflects the percentage of filtered HSP27 that is excreted. Lebherz-Eichinger et al. demonstrated increased fractional HSP27 urine excretion in severe kidney injury (33). Patients included in our cohort did not suffer from kidney injury and did not observe a difference in eGFR in both survivors and decedents (Figure 1C) as well as in fractional HSP27 urine excretion low or high group. Increased fractional
Traxler et al. HSP27 urine excretion in acute exacerbation of COPD

HSP27 urine excretion in patients suffering from kidney injury may be a result of direct release of the failing kidney. Thus, we hypothesize that increasing HSP27 urine excretion and simultaneously decreasing serum and urine HSP27 concentrations after an exacerbation represent less production of HSP27 due to decreasing stress and successful renal elimination of HSP27. This dynamic may reflect a persistent stable condition of those patients.

Additionally to increased HSP27 serum concentration in COPD patients compared to healthy smokers and non-smokers Hacker et al. demonstrated also continuous increase with disease severity (24). In accordance with these findings, we could detect a significant increase of HSP27 urine excretion in GOLD IV patients compared to GOLD II patients. Increased HSP27 urine excretion in GOLD IV patients may reflect augmented excretion by the kidney to eliminate elevated HSP27 production due to increasing stress levels in severely ill patients.

We provide significant evidence that fractional HSP27 urine excretion could serve as a biomarker to identify COPD patients that are at high risk for death within 180 days after discharge after an episode of acute exacerbation with superior performance especially in the first 30 days after discharge in comparison to our already published serum HSP27 based model. Nevertheless, some limitations of our study should be mentioned. First of all, we conducted a single-center study and one must carefully translate our results into other patient groups. Second, we included only GOLD II-IV patients. No intervention apart from standard care was performed so we can only hypothesize that the availability of fractional HSP27 urine excretion to physicians offers a useful tool for the management of AECOPD.

\section{Conclusions}

In conclusion, we found that fractional HSP27 urine excretion is decreased during an episode of acute exacerbation and can serve as a mortality predictor within a short follow up time. We propose that obtaining HSP27 urine and serum concentration at admission and calculating fraction HSP27 urine excretion might offer valuable information for the management of COPD patients admitted to a hospital due to an acute exacerbation within the first year. Whereas fractional HSP27 urine excretion is superior within the first 30 days after discharge, HSP27 serum concentration might provide valuable information for patient management up to 1 year after discharge. 
Nevertheless, further studies including a larger sample size are needed to confirm our results.

\section{Acknowledgments}

Funding: This work was supported by Christian Doppler Laboratory for Cardiac and Thoracic Diagnosis and Regeneration, Medical University of Vienna, Austria.

\section{Footnote}

Reporting Checklist: The authors have completed the STROBE reporting checklist. Available at http://dx.doi. org/10.21037/atm-20-3683

Data Sharing Statement: Available at http://dx.doi. org/10.21037/atm-20-3683

Conflicts of Interest: All authors have completed the ICMJE uniform disclosure form (available at http://dx.doi. org/10.21037/atm-20-3683). MF reports personal fees from Astra Zeneca, personal fees from Boehringer Ingelheim, outside the submitted work. The other authors have no conflicts of interest to declare.

Ethical Statement: The authors are accountable for all aspects of the work in ensuring that questions related to the accuracy or integrity of any part of the work are appropriately investigated and resolved. The study has been approved by the National Ethics Committee of the Republic of Slovenia (EC number: 65/02/09) and conducted according to the Declaration of Helsinki (as revised in 2013). Informed consent was provided by all participants.

Open Access Statement: This is an Open Access article distributed in accordance with the Creative Commons Attribution-NonCommercial-NoDerivs 4.0 International License (CC BY-NC-ND 4.0), which permits the noncommercial replication and distribution of the article with the strict proviso that no changes or edits are made and the original work is properly cited (including links to both the formal publication through the relevant DOI and the license). See: https://creativecommons.org/licenses/by-nc-nd/4.0/.

\section{References}

1. Januzzi JL, Peacock WF, Maisel AS, et al. Measurement of the Interleukin Family Member ST2 in Patients With Acute Dyspnea. J Am Coll Cardiol 2007;50:607-13.

2. Connors AF Jr, Dawson NV, Thomas C, et al. Outcomes following acute exacerbation of severe chronic obstructive lung disease. The SUPPORT investigators (Study to Understand Prognoses and Preferences for Outcomes and Risks of Treatments). Am J Respir Crit Care Med 1996;154:959-67.

3. Wedzicha JA, Seemungal TAR. COPD exacerbations: defining their cause and prevention. Lancet 2007;370:786-96.

4. Hodge G, Mukaro V, Reynolds PN, et al. Role of increased $\mathrm{CD} 8 / \mathrm{CD} 28$ null $\mathrm{T}$ cells and alternative co-stimulatory molecules in chronic obstructive pulmonary disease. Clin Exp Immunol 2011;166:94-102.

5. Lambers $\mathrm{C}$, Hacker $\mathrm{S}$, Posch $\mathrm{M}$, et al. T cell senescence and contraction of $\mathrm{T}$ cell repertoire diversity in patients with chronic obstructive pulmonary disease. Clin Exp Immunol 2009;155:466-75.

6. Hodge G, Roscioli E, Jersmann H, et al. Steroid resistance in COPD is associated with impaired molecular chaperone Hsp90 expression by pro-inflammatory lymphocytes. Respir Res 2016;17:135.

7. MacNee W. Systemic inflammatory biomarkers and comorbidities of chronic obstructive pulmonary disease. Ann Med 2013;45:291-300.

8. Welniak TJ, Panzenbeck A, Koyfman A, et al. Chronic obstructive pulmonary disease: Emergency care in acute exacerbation. Afr J Emerg Med 2015;5:75-84.

9. Oswald-Mammosser M, Weitzenblum E, Quoix E, et al. Prognostic factors in COPD patients receiving long-term oxygen therapy. Importance of pulmonary artery pressure. Chest 1995;107:1193-8.

10. Antonelli Incalzi R, Fuso L, De Rosa M, et al. Comorbidity contributes to predict mortality of patients with chronic obstructive pulmonary disease. Eur Respir J 1997;10:2794-800.

11. Fuso L, Incalzi RA, Pistelli R, et al. Predicting mortality of patients hospitalized for acutely exacerbated chronic obstructive pulmonary disease. Am J Med 1995;98:272-7.

12. Rammaert B, Verdier N, Cavestri B, et al. Procalcitonin as a prognostic factor in severe acute exacerbation of chronic obstructive pulmonary disease. Respirology 2009;14:969-74.

13. Peng C, Tian C, Zhang Y, et al. C-reactive protein levels predict bacterial exacerbation in patients with chronic obstructive pulmonary disease. Am J Med Sci 2013;345:190-4.

14. Bartziokas K, Papaioannou AI, Loukides S, et al. Serum uric acid as a predictor of mortality and future 
Page 12 of 13

exacerbations of COPD. Eur Respir J 2014;43:43-53.

15. Marcun R, Sustic A, Brguljan PM, et al. Cardiac biomarkers predict outcome after hospitalisation for an acute exacerbation of chronic obstructive pulmonary disease. Int J Cardiol 2012;161:156-9.

16. Calderwood SK, Mambula SS, Gray PJ Jr, et al. Extracellular heat shock proteins in cell signaling. FEBS Lett 2007;581:3689-94.

17. Bakthisaran R, Tangirala R, Rao Ch M. Small heat shock proteins: Role in cellular functions and pathology. Biochim Biophys Acta 2015;1854:291-319.

18. Arrigo AP. The cellular "networking" of mammalian Hsp27 and its functions in the control of protein folding, redox state and apoptosis. Adv Exp Med Biol 2007;594:14-26.

19. De AK, Kodys KM, Yeh BS, et al. Exaggerated human monocyte IL-10 concomitant to minimal TNF-alpha induction by heat-shock protein 27 (Hsp27) suggests Hsp27 is primarily an antiinflammatory stimulus. J Immunol 2000;165:3951-8.

20. Rayner K, Chen YX, McNulty M, et al. Extracellular release of the atheroprotective heat shock protein 27 is mediated by estrogen and competitively inhibits acLDL binding to scavenger receptor-A. Circ Res 2008;103:133-41.

21. Ghayour-Mobarhan M, Saber H, Ferns GA. The potential role of heat shock protein 27 in cardiovascular disease. Clin Chim Acta 2012;413:15-24.

22. Lianos GD, Alexiou GA, Mangano A, et al. The role of heat shock proteins in cancer. Cancer Lett 2015;360:114-8.

23. Jan Ankersmit H, Nickl S, Hoeltl E, et al. Increased serum levels of HSP27 as a marker for incipient chronic obstructive pulmonary disease in young smokers. Respiration 2012;83:391-9.

24. Hacker S, Lambers C, Hoetzenecker K, et al. Elevated HSP27, HSP70 and HSP90 alpha in chronic obstructive pulmonary disease: markers for immune activation and tissue destruction. Clin Lab 2009;55:31-40.

25. Cui X, Xing J, Liu Y, et al. COPD and levels of Hsp70 (HSPA1A) and Hsp27 (HSPB1) in plasma and lymphocytes among coal workers: a case-control study. Cell Stress Chaperones 2015;20:473-81.

26. Hu R, Ouyang Q, Dai A, et al. Heat shock protein 27 and cyclophilin A associate with the pathogenesis of COPD. Respirology 2011;16:983-93.

27. Cappello F, Caramori G, Campanella C, et al. Convergent sets of data from in vivo and in vitro methods point to an active role of Hsp60 in chronic obstructive pulmonary disease pathogenesis. PLoS One 2011;6:e28200.
Traxler et al. HSP27 urine excretion in acute exacerbation of COPD

28. Zimmermann M, Traxler D, Bekos C, et al. Heat shock protein 27 as a predictor of prognosis in patients admitted to hospital with acute COPD exacerbation. Cell Stress Chaperones 2020;25:141-9.

29. Corsini E, Galbiati V, Papale A, et al. The role of HSP27 in RACK1-mediated PKC activation in THP-1 cells. Immunol Res 2016;64:940-50.

30. Farkas J, Kadivec S, Kosnik M, et al. Effectiveness of discharge-coordinator intervention in patients with chronic obstructive pulmonary disease, study protocol of a randomized controlled clinical trial. Respir Med 2011;105:S26-30.

31. Lainscak M, Kadivec S, Kosnik M, et al. Discharge coordinator intervention prevents hospitalizations in patients with COPD: a randomized controlled trial. J Am Med Dir Assoc 2013;14:450 e1-6.

32. Zimmermann M, Mueller T, Dieplinger B, et al. Circulating heat shock protein 27 as a biomarker for the differentiation of patients with lung cancer and healthy controls--a clinical comparison of different enzyme linked immunosorbent assays. Clin Lab 2014;60:999-1006.

33. Lebherz-Eichinger D, Ankersmit HJ, Hacker S, et al. HSP27 and HSP70 serum and urine levels in patients suffering from chronic kidney disease. Clin Chim Acta 2012;413:282-6.

34. Cogo A, Ciaccia A, Legorini C, et al. Proteinuria in COPD patients with and without respiratory failure. Chest 2003;123:652-3; author reply 3 .

35. Budczies J, Klauschen F, Sinn BV, et al. Cutoff Finder: a comprehensive and straightforward Web application enabling rapid biomarker cutoff optimization. PLoS One 2012;7:e51862.

36. Brekke PH, Omland T, Holmedal SH, et al. Troponin T elevation and long-term mortality after chronic obstructive pulmonary disease exacerbation. Eur Respir J 2008;31:563-70.

37. Hoiseth AD, Neukamm A, Karlsson BD, et al. Elevated high-sensitivity cardiac troponin $\mathrm{T}$ is associated with increased mortality after acute exacerbation of chronic obstructive pulmonary disease. Thorax 2011;66:775-81.

38. Hoiseth AD, Omland T, Hagve TA, et al. NT-proBNP independently predicts long term mortality after acute exacerbation of COPD - a prospective cohort study. Respir Res 2012;13:97.

39. Medina AM, Marteles MS, Saiz EB, et al. Prognostic utility of NT-proBNP in acute exacerbations of chronic pulmonary diseases. Eur J Intern Med 2011;22:167-71.

40. Chang CL, Robinson SC, Mills GD, et al. Biochemical markers of cardiac dysfunction predict mortality in acute 
exacerbations of COPD. Thorax 2011;66:764-8.

41. Srivastava P. Interaction of heat shock proteins with peptides and antigen presenting cells: chaperoning of the innate and adaptive immune responses. Annu Rev Immunol 2002;20:395-425.

42. Hashiguchi N, Ogura H, Tanaka H, et al. Enhanced expression of heat shock proteins in activated polymorphonuclear leukocytes in patients with sepsis. J Trauma 2001;51:1104-9.

43. Jin C, Phillips VL, Williams MJ, et al. Plasma heat shock protein 27 is associated with coronary artery disease, abdominal aortic aneurysm and peripheral artery disease. Springerplus 2014;3:635.

44. Bernhard D, Rossmann A, Wick G. Metals in cigarette smoke. IUBMB Life 2005;57:805-9.

45. Hawse JR, Cumming JR, Oppermann B, et al. Activation

Cite this article as: Traxler D, Zimmermann M, Simader E, Einwallner E, Copic D, Graf A, Mueller T, Veraar C, Lainscak M, Marčun R, Košnik M, Fležar M, Rozman A, Korošec P, Klepetko W, Moser B, Ankersmit HJ. Fractional heat shock protein 27 urine excretion as a short-term predictor in acute exacerbation of chronic obstructive pulmonary disease. Ann Transl Med 2021;9(2):117. doi: 10.21037/atm-20-3683 of metallothioneins and alpha-crystallin/sHSPs in human lens epithelial cells by specific metals and the metal content of aging clear human lenses. Invest Ophthalmol Vis Sci 2003;44:672-9.

46. van Eeden SF, Sin DD. Oxidative stress in chronic obstructive pulmonary disease: a lung and systemic process. Can Respir J 2013;20:27-9.

47. Merendino AM, Paul C, Vignola AM, et al. Heat shock protein-27 protects human bronchial epithelial cells against oxidative stress-mediated apoptosis: possible implication in asthma. Cell Stress Chaperones 2002;7:269-80.

48. Unver R, Deveci F, Kirkil G, et al. Serum Heat Shock Protein Levels and the Relationship of Heat Shock Proteins with Various Parameters in Chronic Obstructive Pulmonary Disease Patients. Turk Thorac J 2016;17:153-9. 